\title{
Synthesis of axially chiral gold complexes and their applications in asymmetric catalyses
}

\author{
Yin-wei Sun ${ }^{1}$, Qin $\mathrm{Xu}^{* 1}$ and Min Shi ${ }^{* 1,2}$
}

\section{Full Research Paper}

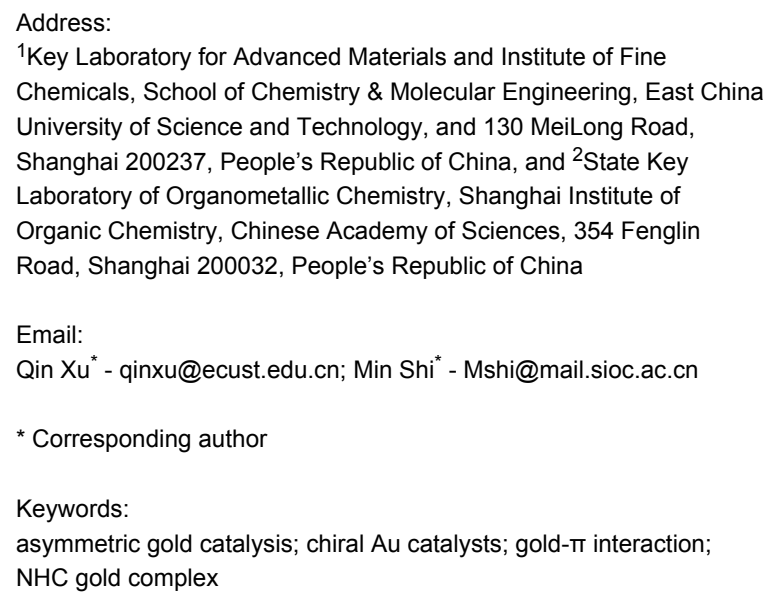

Beilstein J. Org. Chem. 2013, 9, 2224-2232.

doi:10.3762/bjoc. 9.261

Received: 02 July 2013

Accepted: 27 September 2013

Published: 28 October 2013

This article is part of the Thematic Series "Gold catalysis for organic synthesis II".

Guest Editor: F. D. Toste

(c) 2013 Sun et al; licensee Beilstein-Institut.

License and terms: see end of document.

\begin{abstract}
Several novel chiral $N$-heterocyclic carbene and phosphine ligands were prepared from $(S)$-BINOL. Moreover, their ligated Au complexes were also successfully synthesized and characterized by X-ray crystal diffraction. A weak gold- $\pi$ interaction between the $\mathrm{Au}$ atom and the aromatic ring in these gold complexes was identified. Furthermore, we confirmed the formation of a pair of diastereomeric isomers in NHC gold complexes bearing an axially chiral binaphthyl moiety derived from the hindered rotation around $\mathrm{C}-\mathrm{C}$ and $\mathrm{C}-\mathrm{N}$ bonds. In the asymmetric intramolecular hydroamination reaction most of these chiral $\mathrm{Au}(\mathrm{I})$ complexes showed good catalytic activities towards olefins tethered with a NHTs functional group to give the corresponding product in moderate yields and up to $29 \%$ ee.
\end{abstract}

\section{Introduction}

After the long-held assumption of the non-reactivity of gold complexes, numerous reactions catalyzed by gold complexes have emerged in the last 2 decades [1-9]. In the past few years, reports on gold-catalyzed organic transformations have increased substantially [10-29]. Homogeneous gold catalysis has proven to be a powerful tool in organic synthesis. However, chiral gold complexes [30-45], especially chiral NHC-gold complex-catalyzed asymmetric reactions [46-53] are still uncommon. Very few efficient chiral NHC-gold catalysts have been known up to the year of 2013. So far, several axially chiral NHC-gold catalysts based on binaphthyl skeleton such as 1 and 2 [46,49] have been reported with good to excellent chiral inductions in asymmetric gold catalysis (Figure 1). Encouraged by these results, we attempted to develop novel types of axially chiral NHC-gold catalysts based on the binaphthyl skeleton. 
<smiles>CC(C)N(C(C)C)C(Cl)Nc1ccc2ccccc2c1-c1c(Br)ccc2ccccc12</smiles>

$1 \mathrm{Ar}=\mathrm{Ph}, 3,5-\mathrm{CF}_{3} \mathrm{C}_{6} \mathrm{H}_{3}$<smiles>N#Cc1cc2ccccc2c(-c2c(NC(Nc3ccccn3)Nc3ccccn3)c([Al])cc3ccccc23)c1NC(Cl)=[W]</smiles>

$2 \mathrm{Ar}=4-\mathrm{CF}_{3} \mathrm{C}_{6} \mathrm{H}_{4}$

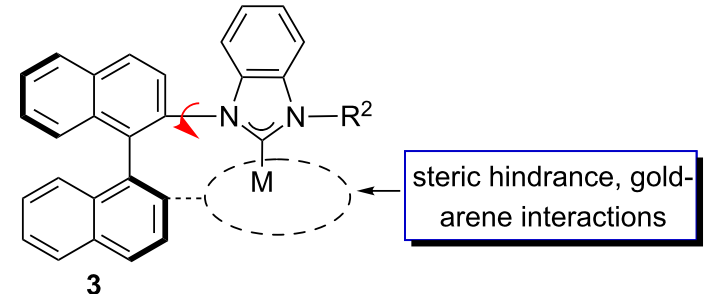

our suggested NHC ligands

Figure 1: Monodentate chiral NHC gold catalysts in recent years.

Very recently, Echavarren's group has reported a very important gold-arene interaction in dialkylbiarylphosphane gold complexes, which is very useful in gold catalysis [54]. It has been disclosed that there was a weak gold- $\pi$ interaction between the gold atom and the aromatic ring in catalysts 1 [46]. On the basis of this finding, we envisaged that if an aryl group is introduced near the ligated gold atom, the gold-arene interaction may affect the catalytic efficiency in gold catalysis (Figure 1).

\section{Results and Discussion}

Synthesis of the carbene-Au(I) complexes. The synthesis of compound 9 was reported by Slaughter and co-workers (Scheme 1) [49]. The usage of $(S)$-BINOL as the starting material to react with trifluoromethanesulfonic anhydride in the presence of DIPEA afforded at $0{ }^{\circ} \mathrm{C}$ in dichloromethane the corresponding product $(S)$-2'-hydroxy-1,1'-binaphthyl-2-yl trifluoromethanesulfonate (5) in good yield. The crude product and $\mathrm{NiCl}_{2}$ (dppe) (10 mol \%) was dissolved in toluene under argon. To this solution was added dropwise a 1.0 M THF solution of 3,5-bis(trifluoromethyl)phenylmagnesium bromide, which afforded $(S)-6$ in 33\% yield in two steps under reflux [55]. Then, $(S)-7$ was obtained by treatment of $(S)-6$ with
$\mathrm{Tf}_{2} \mathrm{O}$ and pyridine in DCM in $99 \%$ yield. The usage of dimethylbis(diphenylphosphino)xanthene (XantPhos) as a ligand and $\mathrm{Pd}_{2}(\mathrm{dba})_{3}$ as a catalyst in the presence of $\mathrm{Cs}_{2} \mathrm{CO}_{3}$, facilitated the reaction of $(S)-7$ with benzylamine in toluene to give the desired compound $(S)-8$ in 57\% yield [49]. Reduction of $(S)-\mathbf{8}$ by using $\mathrm{Pd} / \mathrm{C}$ and $\mathrm{H}_{2}$ in $\mathrm{MeOH}$ produced the desired compound (S)-9 in $95 \%$ yield.

The preparation of chiral benzimidazolium salt $(S)$-13 is shown in Scheme 2. Based on our previous work [52], the coupling reaction between compound ( $S$ )-9 and 1-bromo-2-nitrobenzene was carried out by using $\operatorname{Pd}_{2}(\mathrm{dba})_{3}$ as the catalyst in the presence of bis[2-(diphenylphosphino)phenyl] ether (DPEphos) and $\mathrm{Cs}_{2} \mathrm{CO}_{3}$, affording the desired compound $(S)-\mathbf{1 0}$ in $94 \%$ yield [51]. Reduction of $(S)-\mathbf{1 0}$ was performed under $\mathrm{H}_{2}$ (1.0 atm) atmosphere by using $\mathrm{Pd} / \mathrm{C}$ as a catalyst, giving the desired compound $(S)-11$ in $95 \%$ yield. The subsequent cyclization of $(S)$ 11 with triethyl orthoformate was carried out at $100{ }^{\circ} \mathrm{C}$ in the presence of $p$-toluenesulfonic acid, affording the desired product $(S)-\mathbf{1 2}$ in $89 \%$ yield. The corresponding benzimidazolium salt $(S)$-13 was obtained in quantitative yield upon treating the benzimidazole ring of $(S)$-12 with methyl iodide in acetonitrile<smiles>Oc1ccc2ccccc2c1-c1c(O)ccc2ccccc12</smiles>

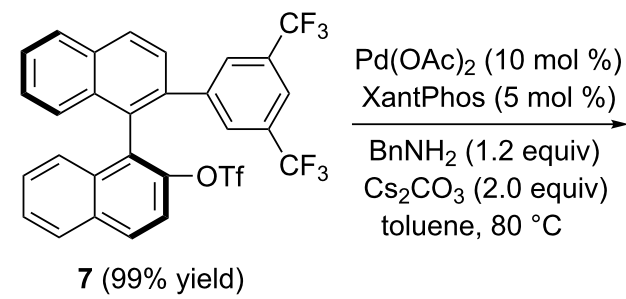

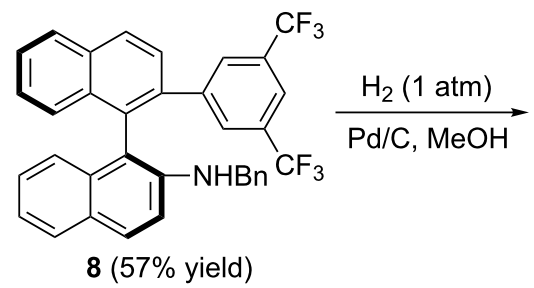<smiles></smiles>

Scheme 1: Synthesis of compound 9. 

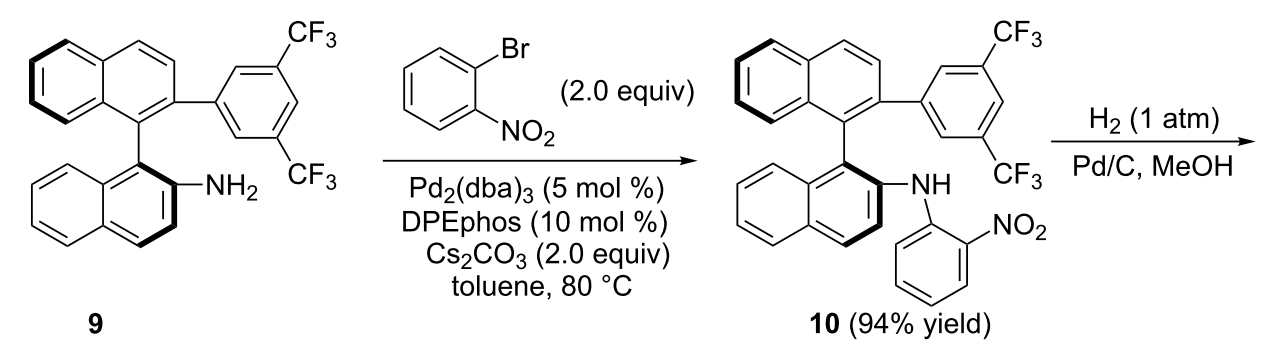

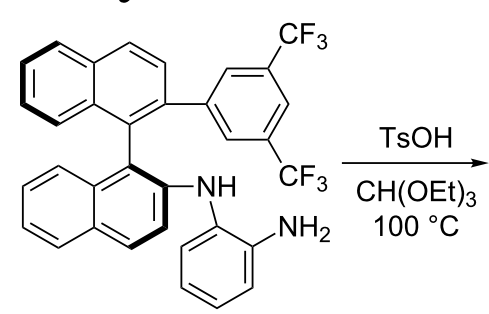

$11(95 \%$ yield $)$

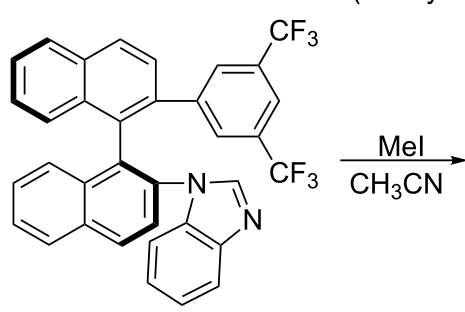

$12(89 \%$ yield $)$

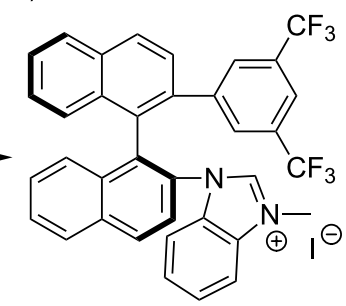

13 (quantitative yield)

Scheme 2: Synthesis of $N$-heterocyclic carbene precursor.

under reflux (Scheme 2). Moreover, treatment of the benzimidazole ring of $(S) \mathbf{- 1 2}$ by using benzyl bromide upon heating in dioxane could produce the corresponding benzimidazolium salt $(S)-14$ also in quantitative yield (Scheme 3 ).

With these NHC precursor salts $(S)-\mathbf{1 3}$ and $(S)$-14 in hand, their coordination pattern with $\mathrm{Au}$ was examined. Benzimidazolium salts $(S)-13$ and $(S)$-14 were treated with $\mathrm{AuCl} \cdot \mathrm{S}(\mathrm{Me})_{2}$ in acetonitrile in the presence of $\mathrm{NaOAc}$ under reflux, giving the corresponding Au complexes $(S)$-15 [two diastereomers: $(S)$-15a in $46 \%$ yield and $(S)-15 b$ in $37 \%$ yield] and $(S)-16$ in $75 \%$ total yield [the two diastereomers: $(S)-\mathbf{1 6 a}$ and $(S)-\mathbf{1 6 b}$ can not be separated by silica gel column chromatography] as a white solid after purification with silica gel column chromatography (Scheme 4). The ratio of $(S)$-16a and $(S)$-16b was identified as 1:2 on the basis of ${ }^{1} \mathrm{H}$ NMR spectroscopic data. After recrystallization from the mixed solvent of DCM and pentane, the single crystals of diastereomers $(S)$-15a and $(S)$-15b were obtained and their structures were confirmed by the X-ray crystal structure diffraction (Figure 2 and Figure 3). The distance between the center of the aromatic ring in one naphthyl moiety (C20-C25) and the $\mathrm{Au}$ atom in $(S)$-15a was only $3.7 \AA$ (Figure 2). The distance from the $\mathrm{Au}$ atom to the center of the bis(trifluoromethyl)phenyl ring (C29-C34) in $(S)$-15b was $3.5 \AA$ (Figure 3). Thus, their X-ray crystal structures clearly revealed the presence of a weak gold- $\pi$ interaction between the Au atom and the aromatic rings in these gold complexes. Because of the gold $-\pi$ interaction, the $\mathrm{C}-\mathrm{N}$ bond could not rotate freely, giving two diastereomeric rotamers $(S)-\mathbf{1 5 a}$ and $(S)-\mathbf{1 5 b}$. Slaughter and co-workers have also found two rotamers in gold complexes $\mathbf{1}$ caused by the handicap of $\mathrm{C}-\mathrm{N}$ bond rotation on the basis of $\mathrm{X}$-ray diffraction and named them as "out" rotamer and "in" rotamer [49] (Scheme 5). Their energy barrier has been also disclosed by DFT calculations.

Synthesis of the $\mathbf{P}-\mathbf{A u}(\mathrm{I})$ complexes. The synthesis of the $\mathrm{Au}$ complexes $(S)$-18 and $(S)$-22 is shown in Scheme 6. Compounds $(S)-\mathbf{1 7}$ and $(S)$-19 were prepared according to published literature procedures [56]. Compound $(S)-17$ was treated with $\mathrm{AuCl} \cdot \mathrm{S}(\mathrm{Me})_{2}$ in acetonitrile at room temperature to give the corresponding Au complex $(S)-\mathbf{1 8}$ in $\mathbf{8 8 \%}$ yield as a white solid after purification with silica gel column chromatography. The

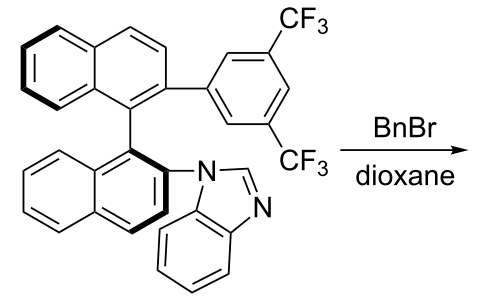

12

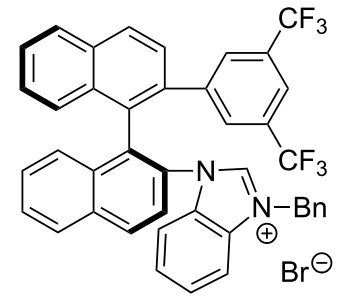

14 (quantitative yield)

Scheme 3: Synthesis of benzimidazolium salt (S)-14 


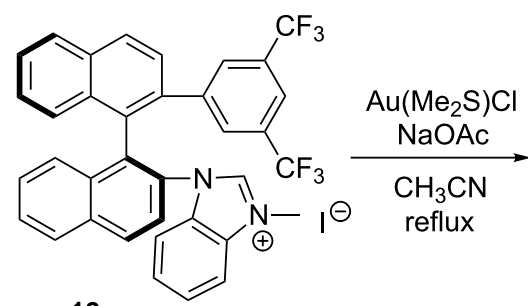

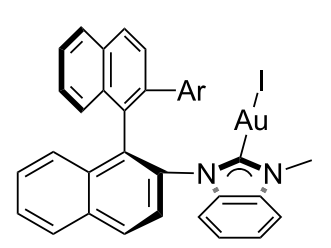

$15 a(46 \%$ yield $)$<smiles>Cn1c([Al]I)[n+](-c2ccc3ccccc3c2-c2c(Br)ccc3ccccc23)c2ccccc21</smiles>

15b (37\% yield) $\mathrm{Ar}=3,5-2 \mathrm{CF}_{3} \mathrm{C}_{6} \mathrm{H}_{3}$<smiles></smiles>

$\mathrm{Ar}=3,5-2 \mathrm{CF}_{3} \mathrm{C}_{6} \mathrm{H}_{3}$

16b $(75 \%$ total yield $)$

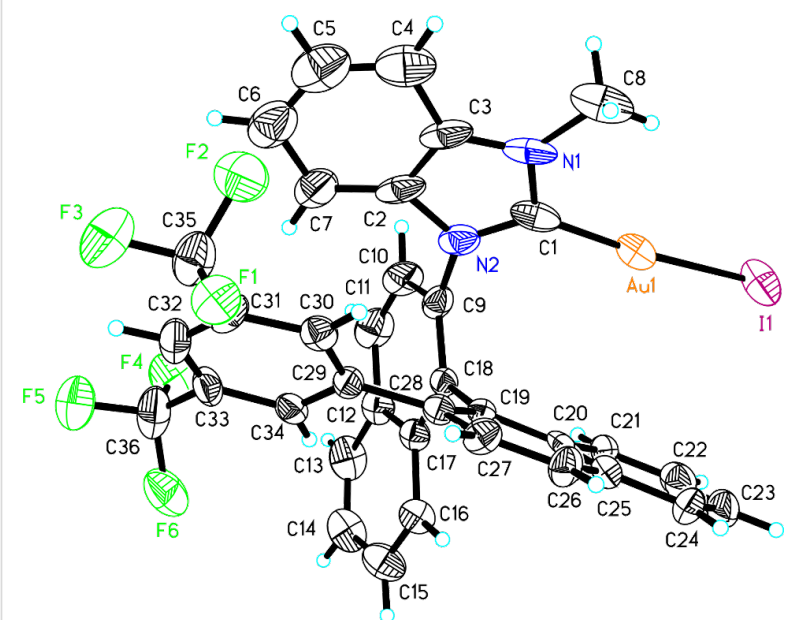

Figure 2: The crystal data of gold complex (S)-15a was deposited in the CCDC with the number 883917. Empirical formula:

$\mathrm{C}_{36} \mathrm{H}_{22} \mathrm{AuF}_{6} \mathrm{IN}_{2}$; formula weight: 920.42 ; crystal color, colorless; crystal dimensions: $0.321 \times 0.212 \times 0.143 \mathrm{~mm}$; crystal system: orthorhombic; lattice parameters: $a=9.6909(5) \AA, b=18.5814(9) \AA$, $c=36.0427(18) \AA, \alpha=90^{\circ}, \beta=90^{\circ}, y=90^{\circ}, V=6490.2(6) \AA^{3}$; space group: $P 2(1) 2(1) 2(1) ; Z=8 ; D_{\text {calc }}=1.884 \mathrm{~g} / \mathrm{cm}^{3} ; F_{000}=3504$; final $R$ indices $[\mathrm{I}>2$ sigma $(\mathrm{I})]$ : R1 $=0.0421 ; \mathrm{wR} 2=0.0793$.

structure of (S)-18 was confirmed by the X-ray crystal structure diffraction (Figure 4). The distance from the $\mathrm{Au}$ atom to the center of the aromatic ring (C11, C12 and C17-C20) in one naphthyl moiety was $3.3 \AA$.

The compound $(S)-19$ and $\mathrm{NiCl}_{2}$ (dppe) (10 mol \%) were dissolved in toluene under argon. To this solution was added dropwise a 1.0 M THF solution of phenylmagnesium bromide and the desired compound $(S)$-20 was afforded in $21 \%$ yield.

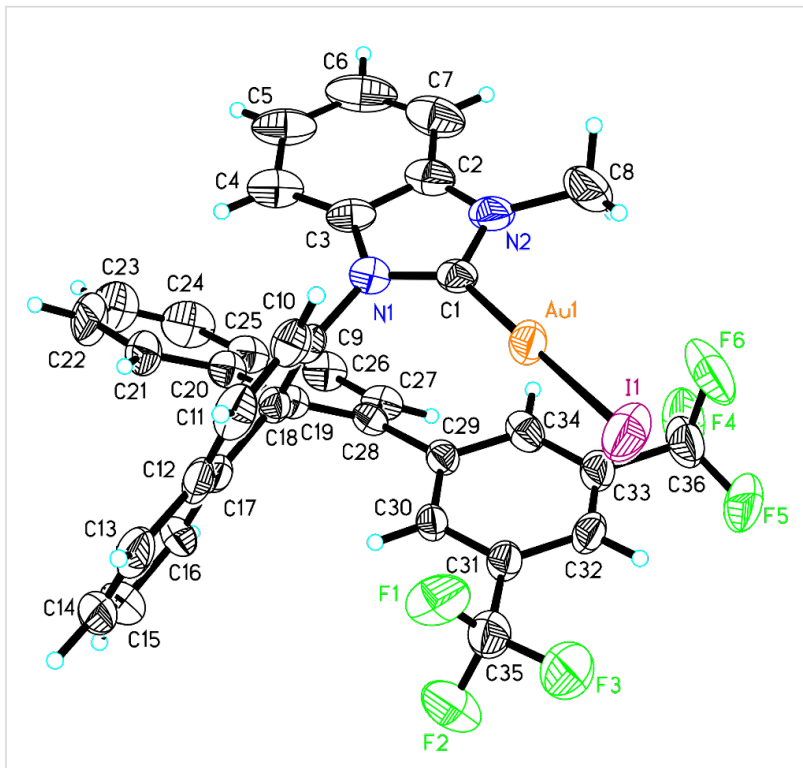

Figure 3: The crystal data of gold complex (S)-15b was deposited in the CCDC with the number 883916. Empirical formula:

$\mathrm{C}_{36} \mathrm{H}_{22} \mathrm{AuF}_{6} \mathrm{IN}_{2}$; formula weight: 920.42 ; crystal color, colorless; crystal dimensions: $0.265 \times 0.211 \times 0.147 \mathrm{~mm}$; crystal system: orthorhombic; lattice parameters: $a=7.6103(5) \AA, b=12.6408(8) \AA$, $c=34.029(2) \AA, \alpha=90^{\circ}, \beta=90^{\circ}, y=90^{\circ}, V=3273.6(4) \AA^{3}$; space group: $P 2(1) 2(1) 2(1) ; Z=4 ; D_{\text {calc }}=1.868 \mathrm{~g} / \mathrm{cm}^{3} ; F_{000}=1752 ;$ final $R$ indices $[\mathrm{I}>2$ sigma $(\mathrm{I})]$ : R1 $=0.0482 ; \mathrm{wR2}=0.1072$.

Then, the obtained compound $(S)$-20 was treated with $\mathrm{SiHCl}_{3}$ in the presence of triethylamine in toluene at $120{ }^{\circ} \mathrm{C}$, giving $(S)$ diphenyl(2'-phenyl-1,1'-binaphthyl-2-yl)phosphine (21) in $81 \%$ yield. The corresponding gold complex $(S)$-22 was obtained in $91 \%$ yield upon treating $(S)$-21 with the same method as the gold complex $(S)$-18. The structure of $(S)$-22 was confirmed by 


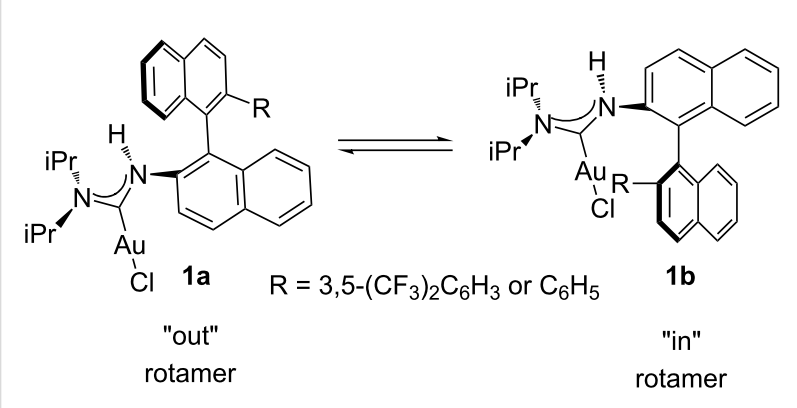

Scheme 5: Rotamers of $\mathbf{1 a}$ and $\mathbf{1 b}$ by DFT calculation reported by Slaughter's group.<smiles>Oc1ccc2ccccc2c1-c1c(P)ccc2ccccc12</smiles>

17<smiles>[PH2+]Oc1ccc2ccccc2c1-c1c([PH2+])ccc2ccccc12</smiles>

19

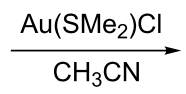<smiles>Oc1ccc2ccccc2c1-c1c([PH+](Cl)Cl)ccc2ccccc12</smiles>

$18(88 \%$ yield $)$

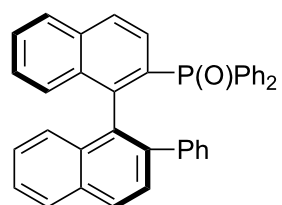

$20(21 \%$ yield $)$<smiles>C[Si](C)(C)[Si](C)(C)[Ge]</smiles>

$\underset{\mathrm{Et}_{3} \mathrm{~N}}{\mathrm{toluene}}$
$120{ }^{\circ} \mathrm{C}$<smiles>Pc1ccccc1-c1ccc2ccccc2c1-c1cccc2ccccc12</smiles>

21 (81\% yield)

Scheme 6: The synthesis of P-Au complexes.

X-ray crystal structure diffraction (Figure 5). The crystal structure of $(S)$-22 (Figure 5) revealed that the distance from the $\mathrm{Au}$ atom to the center of the phenyl ring (C21-C26) was $4.5 \AA$. During the process of the preparation of $(S)$-21, we found a small amount of naphtho[1,2-g]chrysene (23), presumably derived from a cross coupling of compound $(S)$-19 with $\mathrm{PhMgBr}$. Its structure was also confirmed by the X-ray crystal structure diffraction (Figure SI-1 in Supporting Information File 1).

The catalytic activities of these gold complexes were examined by the gold-catalyzed asymmetric intramolecular hydroamination of olefin $\mathbf{2 4}$ tethered with a NHTs functional group.

Intramolecular hydroamination reaction catalyzed by $\mathbf{A u}(\mathbf{I})$ complexes. We synthesized a variety of Au complexes both neutral and cationic and subsequently used these complexes as

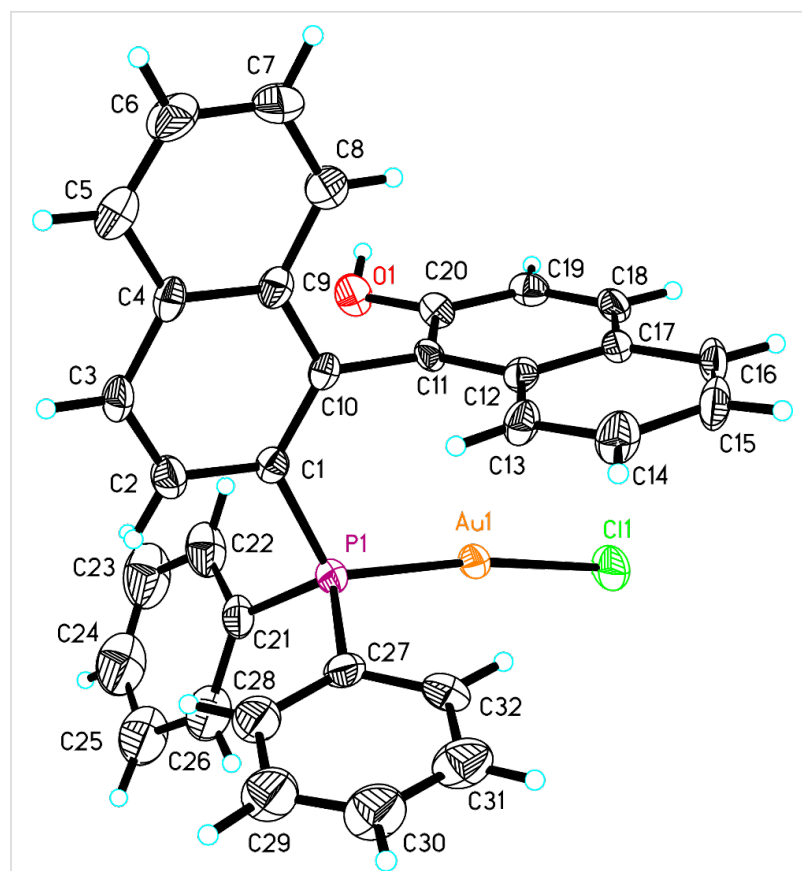

Figure 4: The crystal data of gold complex (S)-18 was deposited in the CCDC with the number 920617. Empirical formula: $\mathrm{C}_{32} \mathrm{H}_{23} \mathrm{AuClOP}$; formula weight: 686.89 ; crystal color, colorless; crystal dimensions: $0.212 \times 0.139 \times 0.101 \mathrm{~mm}$; crystal system: orthorhombic; lattice parameters: $a=9.0411(7) \AA, b=13.4833(10) \AA, c=21.9878(16) \AA$, $\alpha=90^{\circ}, \beta=90^{\circ}, y=90^{\circ}, V=2680.4(3) \AA^{3}$; space group: $P 2(1) 2(1) 2(1) ; Z=4 ; D_{\text {calc }}=1.702 \mathrm{~g} / \mathrm{cm}^{3} ; F_{000}=1336$; final $R$ indices $[\mathrm{I}>2$ sigma $(\mathrm{I})]$ : R1 = 0.0378; $\mathrm{wR} 2=0.0680$.

catalysts in a variety of reactions. High enantioselectivities were achieved in the asymmetric intramolecular hydroamination of allenes by using a variety of chiral phosphine-Au(I) complexes [57-63]. On the other hand, the intramolecular hydroamination of olefins is a more important reaction in organic synthesis and has been widely reported [64-68]. Recently, the enantioselective intramolecular hydroamination of olefins has also been significantly improved by using various transition metal complexes or other metal complexes [69-77]. However, to the best of our knowledge, the enantioselective intramolecular hydroamination of olefins catalyzed by gold complexes has not been reported yet. We therefore applied our Au complexes to the asymmetric catalysis of the intramolecular hydroamination of olefin $\mathbf{2 4}$ tethered with a NHTs functional group.

Treatment of olefin $\mathbf{2 4}$ with the axially chiral gold complex $(S)$ 22 and $\operatorname{AgOTf}(5 \mathrm{~mol} \%)$ in toluene at $85{ }^{\circ} \mathrm{C}$ for $36 \mathrm{~h}$ afforded pyrrolidine derivative 25 in $46 \%$ yield and $17 \%$ ee. While using $\mathrm{AgSbF}_{6}$ or $\mathrm{AgNTf}_{2}$ as additives, only trace amounts of $\mathbf{2 5}$ were formed. Further screening of silver salts revealed that AgOTs showed the best catalytic activity in this reaction, giving $\mathbf{2 5}$ in $72 \%$ yield and $27 \%$ ee (Table 1 , entries $1-6$ ). The usage of other solvents such as DCE, $\mathrm{CH}_{3} \mathrm{CN}$ and THF, decreased significantly the yield (Table 1, entries 7-9). The employment 


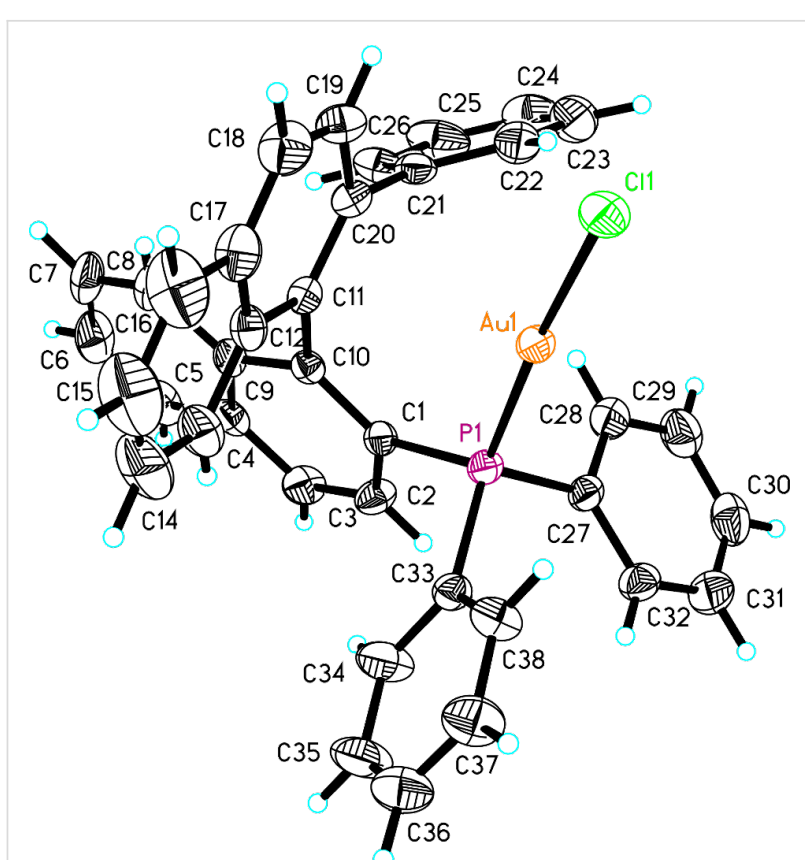

Figure 5: The crystal data of gold complex (S)-22 was deposited in the CCDC with the number 928664. Empirical formula: $\mathrm{C}_{38} \mathrm{H}_{27} \mathrm{AuCIP}$; formula weight: 746.98 ; crystal color, habit: yellow; crystal system: monoclinic; crystal size: $0.21 \times 0.19 \times 0.11$; lattice parameters: $a=10.1476(9) \AA, b=15.7426(14) \AA, c=10.5615(9) \AA, \alpha=90^{\circ}$, $\beta=115.257(2)^{\circ}, y=90^{\circ}, V=1525.9(2) \AA^{3}$; space group: $P 2(1) ; Z=4$; $D_{\text {calc }}=1.626 \mathrm{~g} / \mathrm{cm}^{3} ; \mathrm{F}_{000}=732$; final $\mathrm{R}$ indices $[\mathrm{I}>2$ sigma $(\mathrm{I})]$ : $\mathrm{R} 1=0.0231 ; \mathrm{wR} 2=0.0538$

of other axially chiral Au complexes in this reaction led to similar results, affording 25 in $42-65 \%$ yields and $7-2-7 \%$ ee (Table 1, entries 10-13). The control experiment indicated that no reaction occurred in the absence of a Au catalyst (Table 1, entry 14).

\section{Conclusion}

Axially chiral $\mathrm{Au}(\mathrm{I})$ complexes exhibiting a binaphthalene scaffold with NHC or phosphine gold complexes on one side and an arene moiety on another side were prepared starting from axially chiral BINOL. A weak gold- $\pi$ interaction between the $\mathrm{Au}$ atom and the aromatic ring in these gold complexes was identified. These axially chiral $\mathrm{Au}(\mathrm{I})$ complexes showed moderate catalytic activities along with low chiral inductions in the asymmetric intramolecular hydroamination reaction of olefin $\mathbf{2 4}$ tethered with a functional group of NHT.

\section{Experimental}

\section{Synthesis of NHC-Au(I) complexes (S)-15a and $(S)-15 b$}

The compound $(S)-\mathbf{1 3}(145 \mathrm{mg}, 0.2 \mathrm{mmol})$ and $\mathrm{AuCl} \cdot \mathrm{S}(\mathrm{Me})_{2}$ (59 mg, $0.2 \mathrm{mmol}$ ), NaOAc (33 mg, $0.4 \mathrm{mmol}$ ) were heated under reflux in $\mathrm{CH}_{3} \mathrm{CN}(2 \mathrm{~mL})$ overnight. The volatiles were then removed under reduced pressure and the residue was puri-
Table 1: Asymmetric intramolecular hydroamination catalyzed by Au complexes.
24

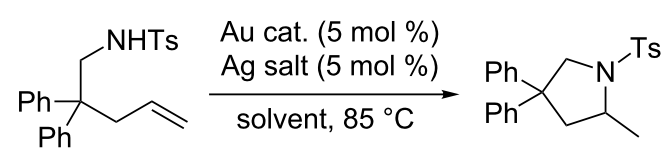

\begin{tabular}{|c|c|c|c|c|c|}
\hline entry ${ }^{a}$ & Au cat. & Ag salt & solvent & yield $(\%)^{b}$ & ee $(\%)^{c}$ \\
\hline 1 & 22 & AgOTf & toluene & 46 & 17 \\
\hline 2 & 22 & $\mathrm{AgSbF}_{6}$ & toluene & trace & $-d$ \\
\hline 3 & 22 & $\begin{array}{c}\mathrm{CF}_{3} \mathrm{CO} \\
\mathrm{OAg}\end{array}$ & toluene & 69 & 11 \\
\hline 4 & 22 & AgOTs & toluene & 72 & 27 \\
\hline 5 & 22 & $\mathrm{AgBF}_{4}$ & toluene & 15 & 10 \\
\hline 6 & 22 & $\mathrm{AgNTf}_{2}$ & toluene & 48 & 0 \\
\hline 7 & 22 & AgOTs & $\mathrm{CH}_{3} \mathrm{CN}$ & 12 & 27 \\
\hline 8 & 22 & AgOTs & DCE & 11 & 29 \\
\hline 9 & 22 & AgOTs & THF & N.R. & $-^{d}$ \\
\hline 10 & 18 & AgOTs & toluene & 58 & 24 \\
\hline 11 & 16 & AgOTs & toluene & 65 & 27 \\
\hline 12 & $15 a$ & AgOTs & toluene & 42 & 7 \\
\hline 13 & $15 b$ & AgOTs & toluene & 51 & 10 \\
\hline 14 & none & AgOTs & toluene & N.R. & $-^{d}$ \\
\hline
\end{tabular}

aThe reaction was carried out on a $0.1 \mathrm{mmol}$ scale in solvents $(1.0 \mathrm{~mL}) .{ }^{\mathrm{b}}$ Isolated yield. ${ }^{\mathrm{c}}$ Measured by chira HPLC. ${ }^{\mathrm{d}}$ Not determined.

fied by a silica gel flash column chromatography to afford goldcomplexes $(S)-\mathbf{1 5 a}(84 \mathrm{mg})$ in $46 \%$ yield and $(S)-\mathbf{1 5 b}(68 \mathrm{mg})$ in $37 \%$ yield. A single crystal grown from complex $(S)-\mathbf{1 5 a}$ or (S)-15b in a saturated solution of $\mathrm{CH}_{2} \mathrm{Cl}_{2}$ /pentane was suitable for X-ray crystal analysis. (S)-15a: white solid; $[\alpha]_{\mathrm{D}}{ }^{20}-64.7(c$ $0.10, \mathrm{CH}_{2} \mathrm{Cl}_{2}$ ); ${ }^{1} \mathrm{H} \mathrm{NMR}\left(400 \mathrm{MHz}, \mathrm{CDCl}_{3}\right.$, TMS) $\delta 8.17-8.13$ (m, 2H, ArH), 7.94 (d, $J=8.8 \mathrm{~Hz}, 1 \mathrm{H}, \mathrm{ArH}), 7.90-7.88$ (m, 1H, ArH), 7.74-7.70 (m, 1H, ArH), 7.66-7.59 (m, 3H, ArH), 7.56-7.50 (m, 2H, ArH), $7.42(\mathrm{~d}, J=8.4 \mathrm{~Hz}, 1 \mathrm{H}, \mathrm{ArH})$, 7.34-7.28 (m, 4H, ArH), 7.06 (s, 2H, ArH), 6.86-6.82 (m, 1H, $\mathrm{ArH}), 5.60(\mathrm{~d}, J=8.4 \mathrm{~Hz}, 1 \mathrm{H}, \mathrm{ArH}), 3.78\left(\mathrm{~s}, 3 \mathrm{H}, \mathrm{CH}_{3}\right)$; ${ }^{19} \mathrm{~F}$ NMR $\left(376 \mathrm{MHz}, \mathrm{CDCl}_{3}, \mathrm{CFCl}_{3}\right) \delta-63.096 ;{ }^{13} \mathrm{C} \mathrm{NMR}$ $\left(100 \mathrm{MHz}, \mathrm{CDCl}_{3}\right) \delta 141.5,140.8,134.9,134.5,133.4,132.9$, 132.6, 131.3, 131.0, 130.9, 130.7, 129.9, 129.2, 129.1, 129.0, $128.5,128.41,128.37,127.9,127.6,127.3,126.9,126.8,126.4$, 123.7, 123.4, 121.0, 120.6, 113.2, 111.4, 34.8; IR $\left(\mathrm{CH}_{2} \mathrm{Cl}_{2}\right)$ v: 3059, 2926, 1594, 1385, 1346, 1277, 1182, 1133, 897, 820, 745, $713 \mathrm{~cm}^{-1}$; HRMS-ESI: $\left[\mathrm{M}+\mathrm{NH}_{4}\right]^{+}$calcd for $\mathrm{C}_{36} \mathrm{H}_{26} \mathrm{AuF}_{6} \mathrm{IN}_{3}$, 938.0736; found, 938.0728. (S)-15b: white solid; $[\alpha]_{\mathrm{D}}{ }^{20}-66.1$ (c $1.45, \mathrm{CH}_{2} \mathrm{Cl}_{2}$ ); ${ }^{1} \mathrm{H}$ NMR (400 MHz, $\mathrm{CDCl}_{3}$, TMS) $\delta 8.17$ (d, $J=8.4 \mathrm{~Hz}, 1 \mathrm{H}, \mathrm{ArH}), 8.13$ (d, $J=8.0 \mathrm{~Hz}, 1 \mathrm{H}, \operatorname{ArH}), 7.85$ (d, $J=8.0 \mathrm{~Hz}, 1 \mathrm{H}, \operatorname{ArH}), 7.79-7.69$ (m, 4H, ArH), 7.63 (d, $J=8.0 \mathrm{~Hz}, 1 \mathrm{H}, \mathrm{ArH}), 7.59-7.54(\mathrm{~m}, 3 \mathrm{H}, \mathrm{ArH}), 7.50-7.46(\mathrm{~m}$, $1 \mathrm{H}, \mathrm{ArH}), 7.23(\mathrm{~d}, J=8.8 \mathrm{~Hz}, 2 \mathrm{H}, \mathrm{ArH}), 7.17$ (s, 2H, ArH), 7.08-7.04 (m, 1H, ArH), 6.47-6.42 (m, 2H, ArH), 3.94 (s, 3H, 
$\left.\mathrm{CH}_{3}\right) ;{ }^{19} \mathrm{~F} \mathrm{NMR}\left(376 \mathrm{MHz}, \mathrm{CDCl}_{3}, \mathrm{CFCl}_{3}\right) \delta-62.451$; ${ }^{13} \mathrm{C}$ NMR $\left(100 \mathrm{MHz}, \mathrm{CDCl}_{3}\right) \delta 134.6,134.5,134.1,134.0$, $133.6,131.4,131.34,131.27,129.1,129.0,128.8,128.7$, $128.66,128.58,128.5,128.3,128.2,127.8,126.9,126.5,124.1$, 123.4, 118.6, 31.9; IR $\left(\mathrm{CH}_{2} \mathrm{Cl}_{2}\right)$ v: 2923, 2851, 1726, 1465, $1387,1277,1181,1131,894,823,743,712,681 \mathrm{~cm}^{-1}$; HRMS-ESI: $\left[\mathrm{M}+\mathrm{NH}_{4}\right]^{+}$calcd for $\mathrm{C}_{36} \mathrm{H}_{26} \mathrm{AuF}_{6} \mathrm{IN}_{3}, 938.0736$; found, 938.0725 .

\section{Synthesis of chiral P-Au(I) complexes (S)-18 and $(S)-22$}

The compound $(S)-17(454 \mathrm{mg}, 1.0 \mathrm{mmol})$ and $\mathrm{AuCl} \cdot \mathrm{S}(\mathrm{Me})_{2}$ (294 mg, $1.0 \mathrm{mmol}$ ) were stirred in $\mathrm{CH}_{3} \mathrm{CN}(10 \mathrm{~mL})$ overnight. The volatiles were then removed under reduced pressure and the residue was purified by silica gel flash column chromatography to afford gold-complex $(S)-\mathbf{1 8}(603 \mathrm{mg})$ in $88 \%$ yield. A single crystal grown from complex $(S)-\mathbf{1 8}$ in a saturated solution of $\mathrm{CH}_{2} \mathrm{Cl}_{2}$ /pentane was suitable for X-ray crystal analysis. (S)-18: white solid; $[\alpha]_{\mathrm{D}}{ }^{20}-35.4\left(c 0.20, \mathrm{CH}_{2} \mathrm{Cl}_{2}\right) ;{ }^{1} \mathrm{H}$ NMR (400 MHz, $\mathrm{CDCl}_{3}$, TMS) $\delta$ 7.99-7.93 (m, 3H, ArH), 7.80 (d, $J=8.4 \mathrm{~Hz}, 1 \mathrm{H}, \mathrm{ArH}), 7.60-7.56(\mathrm{~m}, 1 \mathrm{H}, \mathrm{ArH}), 7.50-7.45(\mathrm{~m}$, $3 \mathrm{H}, \mathrm{ArH}), 7.42-7.17$ (m, 12H, ArH), 6.86-6.82 (m, 1H, ArH), $6.45(\mathrm{~d}, J=8.4 \mathrm{~Hz}, 1 \mathrm{H}, \mathrm{ArH}), 5.17$ (br, 1H, OH); ${ }^{31} \mathrm{P}$ NMR (162 $\mathrm{MHz}, \mathrm{CDCl}_{3}, 85 \% \mathrm{H}_{3} \mathrm{PO}_{4}$ ) $\delta 26.116 ;{ }^{13} \mathrm{C} \mathrm{NMR}$ $\left(100 \mathrm{MHz}, \mathrm{CDCl}_{3}\right) \delta 141.8,136.5,134.4,133.7,133.24$, $133.22,133.1,132.39,132.35,130.5,130.2,129.8,129.0$, $128.9,128.6,128.4,127.6,127.3,127.2,127.1,127.0,126.6$, 124.2, 123.7, 123.0, 112.6, 110.4; IR $\left(\mathrm{CH}_{2} \mathrm{Cl}_{2}\right)$ v: 3359, 3055, 2924, 1623, 1513, 1435, 1269, 1098, 972, 937, 814, 743, 692 $\mathrm{cm}^{-1}$; HRMS-ESI: $\left[\mathrm{M}+\mathrm{NH}_{4}\right]^{+}$: calcd for $\mathrm{C}_{32} \mathrm{H}_{27} \mathrm{AuClNOP}$, 704.1179; found, 704.1170.

Gold complex $(S)$-22 has been prepared by the same reaction procedure as gold complex $(S)-\mathbf{1 8}$ in $91 \%$ yield. A single crystal grown from complex $(S)-22$ in a saturated solution of $\mathrm{CH}_{2} \mathrm{Cl}_{2}$ / pentane was suitable for X-ray crystal analysis. white solid; $[\alpha]_{\mathrm{D}}{ }^{20}-80.7\left(c 0.95, \mathrm{CH}_{2} \mathrm{Cl}_{2}\right) ;{ }^{1} \mathrm{H} \mathrm{NMR}\left(400 \mathrm{MHz}, \mathrm{CDCl}_{3}\right.$, TMS) $\delta 8.27(\mathrm{~d}, J=8.8 \mathrm{~Hz}, 1 \mathrm{H}, \mathrm{ArH}), 8.05(\mathrm{~d}, J=8.4 \mathrm{~Hz}, 1 \mathrm{H}$, $\operatorname{ArH}), 7.90(\mathrm{~d}, J=8.0 \mathrm{~Hz}, 1 \mathrm{H}, \operatorname{ArH}), 7.84(\mathrm{~d}, J=8.4 \mathrm{~Hz}, 1 \mathrm{H}$, $\operatorname{ArH}), 7.67$ (d, $J=8.4 \mathrm{~Hz}, 1 \mathrm{H}, \mathrm{ArH}), 7.62-7.57$ (m, 1H, ArH), $7.47(\mathrm{t}, J=7.6 \mathrm{~Hz}, 1 \mathrm{H}, \mathrm{ArH}), 7.40-7.35$ (m, 4H, ArH), 7.28-7.24 (m, 2H, ArH), 7.22-7.12 (m, 6H, ArH), 6.97 (d, $J=7.6 \mathrm{~Hz}, 2 \mathrm{H}, \operatorname{ArH}), 6.92(\mathrm{t}, J=7.2 \mathrm{~Hz}, 2 \mathrm{H}, \operatorname{ArH}), 6.88$ (d, $J=7.6 \mathrm{~Hz}, 1 \mathrm{H}, \mathrm{ArH}), 6.84(\mathrm{~d}, J=8.8 \mathrm{~Hz}, 1 \mathrm{H}, \mathrm{ArH}), 6.77(\mathrm{t}$, $J=7.6 \mathrm{~Hz}, 2 \mathrm{H}, \mathrm{ArH}) ;{ }^{31} \mathrm{P} \mathrm{NMR}\left(162 \mathrm{MHz}, \mathrm{CDCl}_{3}, 85 \%\right.$ $\left.\mathrm{H}_{3} \mathrm{PO}_{4}\right) \delta 22.898,22.825,22.751,22.697 ;{ }^{13} \mathrm{C} \mathrm{NMR}(100 \mathrm{MHz}$, $\left.\mathrm{CDCl}_{3}\right) \delta 151.4,134.6,134.4,134.1,133.9,133.7,133.5$, $133.2,133.1,131.5,131.44,131.37,131.35,131.25,129.9$, $129.3,129.04,128.98,128.92,128.89,128.87,128.85,128.84$, $128.7,128.64,128.59,128.51,128.4,128.3,128.2,127.8$, $127.5,126.81,126.80,126.6,126.5,126.47,126.1,126.0$,
124.1, 123.4; IR $\left(\mathrm{CH}_{2} \mathrm{Cl}_{2}\right)$ v: 3054, 1589, 1494, 1480, 1436, 1306, 1265, 1098, 1027, 819, 763, 744, $698 \mathrm{~cm}^{-1}$; HRMS-ESI: $\left[\mathrm{M}+\mathrm{NH}_{4}\right]^{+}$calcd for $\mathrm{C}_{38} \mathrm{H}_{31} \mathrm{AuClNP}, 764.1543$; found, 764.1532 .

\section{General procedure for the intramolecular hydroamination reaction catalyzed by $\mathrm{Au}(\mathrm{I})$ complexes}

In a similar way as described in reference [51], a mixture of $\mathrm{Au}$ catalyst (5 mol \%) and $\operatorname{AgX}(5 \mathrm{~mol} \%)$ in solvent $(0.5 \mathrm{~mL})$ was stirred at room temperature for $5 \mathrm{~min}$ under argon, then a solution of compound $24(39.1 \mathrm{mg}, 0.10 \mathrm{mmol})$ in solvent $(0.5 \mathrm{~mL})$ was added into the resulting solution. The resulting suspension was stirred at $85{ }^{\circ} \mathrm{C}$ for $36 \mathrm{~h}$. Column chromatography of the reaction mixture gave the desired product. The enantiomeric purity of the product was determined by chiral HPLC analysis. Compound 25: ${ }^{1} \mathrm{H}$ NMR (400 MHz, $\left.\mathrm{CDCl}_{3}, \mathrm{TMS}\right) \delta 7.61$ (d, $J=8.0 \mathrm{~Hz}, 2 \mathrm{H}, \mathrm{ArH}), 7.27-7.09$ (m, 12H, ArH), 4.17 (d, $\left.J=10.4 \mathrm{~Hz}, 1 \mathrm{H}, \mathrm{CH}_{2}\right), 3.94\left(\mathrm{dd}, J_{1}=10.4 \mathrm{~Hz}, J_{2}=0.4 \mathrm{~Hz}, 1 \mathrm{H}\right.$, $\left.\mathrm{CH}_{2}\right), 3.82-3.74(\mathrm{~m}, 1 \mathrm{H}, \mathrm{CH}), 2.78\left(\mathrm{ddd}, J_{1}=12.4 \mathrm{~Hz}\right.$, $\left.J_{2}=7.2 \mathrm{~Hz}, J_{3}=0.4 \mathrm{~Hz}, 1 \mathrm{H}, \mathrm{CH}_{2}\right), 2.38\left(\mathrm{~s}, 3 \mathrm{H}, \mathrm{CH}_{3}\right), 2.26(\mathrm{dd}$, $\left.J_{1}=12.4 \mathrm{~Hz}, J_{2}=7.2 \mathrm{~Hz}, 1 \mathrm{H}, \mathrm{CH}_{2}\right), 1.25(\mathrm{~d}, J=6.4 \mathrm{~Hz}, 3 \mathrm{H}$, $\left.\mathrm{CH}_{3}\right) ;{ }^{13} \mathrm{C}$ NMR $\left(100 \mathrm{MHz}, \mathrm{CDCl}_{3}\right) \delta 145.6,144.8,142.9$, $135.3,129.5,128.43,128.42,127.1,126.6,126.42,126.39$, 126.2, 58.3, 55.4, 52.2, 45.9, 22.1, 21.4; $[\alpha]_{\mathrm{D}}{ }^{20} 20.1$ (c 1.2, $\mathrm{CH}_{2} \mathrm{Cl}_{2}$ ), for $29 \%$ ee; Chiralcel PA-2, hexane/iPrOH $=60 / 40$, $0.5 \mathrm{~mL} / \mathrm{min}, 214 \mathrm{~nm}, t_{\text {major }}=45.07 \mathrm{~min}, t_{\text {minor }}=27.49 \mathrm{~min}$.

\section{Supporting Information}

\section{Supporting Information File 1}

Experimental procedures and characterization date of compounds.

[http://www.beilstein-journals.org/bjoc/content/ supplementary/1860-5397-9-261-S1.pdf]

\section{Supporting Information File 2}

Chemical information file of compound $(S)$-15a. [http://www.beilstein-journals.org/bjoc/content/ supplementary/1860-5397-9-261-S2.cif]

\section{Supporting Information File 3}

Chemical information file of compound $(S)$-15b. [http://www.beilstein-journals.org/bjoc/content/ supplementary/1860-5397-9-261-S3.cif]

\section{Supporting Information File 4}

Chemical information file of compound $(S)-\mathbf{1 8}$. [http://www.beilstein-journals.org/bjoc/content/ supplementary/1860-5397-9-261-S4.cif] 


\section{Supporting Information File 5}

Chemical information file of compound $(S)-\mathbf{2 1}$.

[http://www.beilstein-journals.org/bjoc/content/

supplementary/1860-5397-9-261-S5.cif]

\section{Supporting Information File 6}

Chemical information file of compound $(S)-\mathbf{2 3}$.

[http://www.beilstein-journals.org/bjoc/content/

supplementary/1860-5397-9-261-S6.cif]

\section{Acknowledgements}

Financial support from the Shanghai Municipal Committee of Science and Technology (08dj1400100-2), the Fundamental Research Funds for the Central Universities, the National Basic Research Program of China (973)-2010CB833302, the Fundamental Research Funds for the Central Universities, and the National Natural Science Foundation of China (21072206, 21121062, 21121062, 20902019, 20472096, 20872162, 20672127, 20732008, 20821002, and 20702013) are gratefully acknowledged.

\section{References}

1. Corma, A.; Leyva-Pérez, A.; Sabater, M. J. Chem. Rev. 2011, 111, 1657-1712. doi:10.1021/cr100414u

2. Li, Z.; Brouwer, C.; He, C. Chem. Rev. 2008, 108, 3239-3265. doi:10.1021/cr068434I

3. Krause, N.; Winter, C. Chem. Rev. 2011, 111, 1994-2009. doi:10.1021/cr1004088

4. Nolan, S. P. Acc. Chem. Res. 2011, 44, 91-100. doi:10.1021/ar1000764

5. Gaillard, S.; Cazin, C. S. J.; Nolan, S. P. Acc. Chem. Res. 2012, 45, 778-787. doi:10.1021/ar200188f

6. Rudolph, M.; Hashmi, A. S. K. Chem. Soc. Rev. 2012, 41, 2448-2462. doi:10.1039/C1CS15279C

7. Wegner, H. A.; Auzias, M. Angew. Chem., Int. Ed. 2011, 50, 8236-8247. doi:10.1002/anie.201101603

8. Huang, H.; Zhou, Y.; Liu, H. Beilstein J. Org. Chem. 2011, 7, 897-936. doi:10.3762/bjoc.7.103

9. Bandini, M. Chem. Soc. Rev. 2011, 40, 1358-1367. doi:10.1039/C0CS00041H

10. Cheon, C. H.; Kanno, O.; Toste, F. D. J. Am. Chem. Soc. 2011, 133, 13248-13251. doi:10.1021/ja204331w

11. Sethofer, S. G.; Mayer, T.; Toste, F. D. J. Am. Chem. Soc. 2010, 132, 8276-8277. doi:10.1021/ja103544p

12. Rudolph, M. Applications of Gold-Catalyzed Reactions to Natural Product Synthesis. In Modern Gold Catalyzed Synthesis; Hashmi, A. S. K.; Toste, F. D., Eds.; Wiley-VCH: Weinheim, Germany, 2012; pp 331-362.

13. Hubbert, C.; Hasmi, A. S. K. Gold-Catalyzed Aldol and Related Reactions. In Modern Gold Catalyzed Synthesis; Hashmi, A. S. K.; Toste, F. D., Eds.; Wiley-VCH: Weinheim, Germany, 2012; pp 237-261.
14. Shapiro, N. D.; Toste, F. D. Synlett 2010, 675-691. doi:10.1055/s-0029-1219369

15. Melhado, A. D.; Brenzovich, W. E., Jr.; Lackner, A. D.; Toste, F. D. J. Am. Chem. Soc. 2010, 132, 8885-8887. doi:10.1021/ja1034123

16. Benitez, D.; Tkatchouk, E.; Gonzalez, A. Z.; Goddard, W. A., III; Toste, F. D. Org. Lett. 2009, 11, 4798-4801. doi:10.1021/ol9018002

17. Shapiro, N. D.; Shi, Y.; Toste, F. D. J. Am. Chem. Soc. 2009, 131, 11654-11655. doi:10.1021/ja903863b

18. Tkatchouk, E.; Mankad, N. P.; Benitez, D.; Goddard, W. A., III; Toste, F. D. J. Am. Chem. Soc. 2011, 133, 14293-14300. doi:10.1021/ja2012627

19. Mauleón, P.; Zeldin, R. M.; González, A. Z.; Toste, F. D. J. Am. Chem. Soc. 2009, 131, 6348-6349. doi:10.1021/ja901649s

20. Hashmi, A. S. K.; Hengst, T.; Lothschütz, C.; Rominger, F. Adv. Synth. Catal. 2010, 352, 1315-1337. doi:10.1002/adsc. 201000126

21. Kusama, H.; Karibe, Y.; Onizawa, Y.; Iwasawa, N. Angew. Chem., Int. Ed. 2010, 49, 4269-4272. doi:10.1002/anie.201001061

22. Teng, T.-M.; Liu, R.-S. J. Am. Chem. Soc. 2010, 132, 9298-9300. doi:10.1021/ja1043837

23. Mukherjee, A.; Dateer, R. B.; Chaudhuri, R.; Bhunia, S.; Karad, S. N.; Liu, R.-S. J. Am. Chem. Soc. 2011, 133, 15372-15375. doi:10.1021/ja208150d

24. Jurberg, I. D.; Odabachian, Y.; Gagosz, F. J. Am. Chem. Soc. 2010, 132, 3543-3552. doi:10.1021/ja9100134

25. Bolte, B.; Gagosz, F. J. Am. Chem. Soc. 2011, 133, 7696-7699. doi:10.1021/ja202336p

26. Ye, L.; Wang, Y.; Aue, D. H.; Zhang, L. J. Am. Chem. Soc. 2012, 134, 31-34. doi:10.1021/ja2091992

27. Hashmi, A. S. K.; Braun, I.; Nösel, P.; Schädlich, J.; Wieteck, M.; Rudolph, M.; Rominger, F. Angew. Chem., Int. Ed. 2012, 51, 4456-4460. doi:10.1002/anie.201109183

28. Barluenga, J.; Sigüeiro, R.; Vicente, R.; Ballesteros, A.; Tomás, M.; Rodríguez, M. A. Angew. Chem., Int. Ed. 2012, 51, 10377-10381. doi:10.1002/anie.201205051

29. Mukherjee, P.; Widenhoefer, R. A. Chem.-Eur. J. 2013, 19, 3437-3444. doi:10.1002/chem.201203987

30. Melhado, A. D.; Amarante, G. W.; Wang, Z. J.; Luparia, M.; Toste, F. D. J. Am. Chem. Soc. 2011, 133, 3517-3527. doi:10.1021/ja1095045

31. Liu, B.; Li, K.-N.; Luo, S.-W.; Huang, J.-Z.; Pang, H.; Gong, L.-Z. J. Am. Chem. Soc. 2013, 135, 3323-3326. doi:10.1021/ja3110472

32. Liu, X.-Y.; Che, C.-M. Org. Lett. 2009, 11, 4204-4207. doi:10.1021/ol901443b

33. Alonso, I.; Trillo, B.; López, F.; Montserrat, S.; Ujaque, G.; Castedo, L.; Lledós, A.; Mascareñas, J. L. J. Am. Chem. Soc. 2009, 131 13020-13030. doi:10.1021/ja905415r

34. Briones, J. F.; Davies, H. M. L. J. Am. Chem. Soc. 2012, 134, 11916-11919. doi:10.1021/ja304506g

35. González, A. Z.; Toste, F. D. Org. Lett. 2010, 12, 200-203. doi:10.1021/ol902622b

36. González, A. Z.; Benitez, D.; Tkatchouk, E.; Goddard, W. A., III; Toste, F. D. J. Am. Chem. Soc. 2011, 133, 5500-5507. doi:10.1021/ja200084a

37. Rodríguez, L.-I.; Roth, T.; Fillol, J. L.; Wadepohl, H.; Gade, L. H. Chem.-Eur. J. 2012, 18, 3721-3728. doi:10.1002/chem.201103140

38. Qian, D.; Zhang, J. Chem.-Eur. J. 2013, 19, 6984-6988. doi:10.1002/chem.201301208

39. Ye, L.; He, W.; Zhang, L. Angew. Chem., Int. Ed. 2011, 50, 3236-3239. doi:10.1002/anie.201007624 
40. Suárez-Pantiga, S.; Hernández-Díaz, C.; Rubio, E.; González, J. M. Angew. Chem., Int. Ed. 2012, 51, 11552-11555.

doi:10.1002/anie.201206461

41. Liu, F.; Qian, D.; Li, L.; Zhao, X.; Zhang, J. Angew. Chem., Int. Ed. 2010, 49, 6669-6672. doi:10.1002/anie.201003136

42. Bandini, M.; Eichholzer, A. Angew. Chem., Int. Ed. 2009, 48, 9533-9537. doi:10.1002/anie.200904388

43. Faustino, H.; Alonso, I.; Mascareñas, J. L.; López, F. Angew. Chem., Int. Ed. 2013, 52, 6526-6530. doi:10.1002/anie.201302713

44. Butler, K. L.; Tragni, M.; Widenhoefer, R. A. Angew. Chem., Int. Ed. 2012, 51, 5175-5178. doi:10.1002/anie.201201584

45. Alonso, I.; Faustino, H.; López, F.; Mascareñas, J. L. Angew. Chem., Int. Ed. 2011, 50, 11496-11500. doi:10.1002/anie.201105815

46. Wang, Y.-M.; Kuzniewski, C. N.; Rauniyar, V.; Hoong, C.; Toste, F. D. J. Am. Chem. Soc. 2011, 133, 12972-12975. doi:10.1021/ja205068j

47. Alcarazo, M.; Stork, T.; Anoop, A.; Thiel, W.; Fürstner, A. Angew. Chem., Int. Ed. 2010, 49, 2542-2546. doi:10.1002/anie.200907194

48. Francos, J.; Grande-Carmona, F.; Faustino, H.; Iglesias-Sigüenza, J.; Díez, E.; Alonso, I.; Fernández, R.; Lassaletta, J. M.; López, F.; Mascareñas, J. L. J. Am. Chem. Soc. 2012, 134, 14322-14325. doi:10.1021/ja3065446

49. Handa, S.; Slaughter, L. M. Angew. Chem., Int. Ed. 2012, 51, 2912-2915. doi:10.1002/anie.201107789

50. Yang, J.; Zhang, R.; Wang, W.; Zhang, Z.; Shi, M. Tetrahedron: Asymmetry 2011, 22, 2029-2038. doi:10.1016/j.tetasy.2011.12.004

51. Wang, W.; Yang, J.; Wang, F.; Shi, M. Organometallics 2011, 30 , 3859-3869. doi:10.1021/om2004404

52. Liu, L.; Wang, F.; Wang, W.; Zhao, M.; Shi, M. Beilstein J. Org. Chem. 2011, 7, 555-564. doi:10.3762/bjoc.7.64

53. Wang, F.; Li, S.; Qu, M.; Zhao, M.; Liu, L.; Shi, M. Beilstein J. Org. Chem. 2012, 8, 726-731. doi:10.3762/bjoc.8.81

54. Pérez-Galán, P.; Delpont, N.; Herrero-Gómez, E.; Maseras, F.; Echavarren, A. M. Chem.-Eur. J. 2010, 16, 5324-5332. doi:10.1002/chem.200903507

55. Ooi, T.; Ohmatsu, K.; Maruoka, K. J. Am. Chem. Soc. 2007, 129, 2410-2411. doi:10.1021/ja063051q

56. Jiang, Y.-Q.; Shi, Y.-L.; Shi, M. J. Am. Chem. Soc. 2008, 130, 7202-7203. doi:10.1021/ja802422d

57. Teller, H.; Corbet, M.; Mantilli, L.; Gopakumar, G.; Goddard, R.; Thiel, W.; Fürstner, A. J. Am. Chem. Soc. 2012, 134, 15331-15342. doi:10.1021/ja303641p

58. Kim, J. H.; Park, S.-W.; Park, S. R.; Lee, S.; Kang, E. J. Chem.-Asian J. 2011, 6, 1982-1986. doi:10.1002/asia.201100135

59. Li, H.; Lee, S. D.; Widenhoefer, R. A. J. Organomet. Chem. 2011, 696, 316-320. doi:10.1016/j.jorganchem.2010.09.045

60. Bartolome, C.; García-Cuadrado, D.; Ramiro, Z.; Espinet, P. Organometallics 2010, 29, 3589-3592. doi:10.1021/om100507r

61. Zhang, Z.; Bender, C. F.; Widenhoefer, R. A. J. Am. Chem. Soc. 2007, 129, 14148-14149. doi:10.1021/ja0760731

62. Zhang, Z.; Bender, C. F.; Widenhoefer, R. A. Org. Lett. 2007, 9 , 2887-2889. doi:10.1021/ol071108n

63. LaLonde, R. L.; Sherry, B. D.; Kang, E. J.; Toste, F. D. J. Am. Chem. Soc. 2007, 129, 2452-2453. doi:10.1021/ja068819|

64. Kitahara, H.; Sakurai, H. J. Organomet. Chem. 2011, 442-449. doi:10.1016/j.jorganchem.2010.08.038
65.Zhang, R.; Xu, Q.; Mei, L.-y.; Li, S.-k.; Shi, M. Tetrahedron 2012, 68, 3172-3178. doi:10.1016/j.tet.2012.02.060

66. Li, Z.; Zhang, J.; Brouwer, C.; Yang, C.-G.; Reich, N. W.; He, C. Org. Lett. 2006, 8, 4175-4178. doi:10.1021/ol0610035

67.Zhang, J.; Yang, C.-G.; He, C. J. Am. Chem. Soc. 2006, 128, 1798-1799. doi:10.1021/ja053864z

68. Kitahara, H.; Kamiya, I.; Sakurai, H. Chem. Lett. 2009, 38, 908-909. doi:10.1246/cl.2009.908

69. Reznichenko, A. L.; Hultzsch, K. C. Organometallics 2013, 32, 1394-1408. doi:10.1021/om3010614

70. Turnpenny, B. W.; Hyman, K. L.; Chemler, S. R. Organometallics 2012 , 31, 7819-7822. doi:10.1021/om300744m

71. Chapurina, Y.; Ibrahim, H.; Guillot, R.; Kolodziej, E.; Collin, J.; Trifonov, A.; Schulz, E.; Hannedouche, J. J. Org. Chem. 2011, 76, 10163-10172. doi:10.1021/jo202009q

72. Manna, K.; Xu, S.; Sadow, A. D. Angew. Chem., Int. Ed. 2011, 50, 1865-1868. doi:10.1002/anie.201006163

73. Ayinla, R. O.; Gibson, T.; Schafer, L. L. J. Organomet. Chem. 2010, 696, 50-60. doi:10.1016/j.jorganchem.2010.07.023

74. Hannedouche, J.; Collin, J.; Trifonov, A.; Schulz, E. J. Organomet. Chem. 2011, 696, 255-262. doi:10.1016/j.jorganchem.2010.09.013

75. Shen, X.; Buchwald, S. L. Angew. Chem., Int. Ed. 2010, 49, 564-567. doi:10.1002/anie.200905402

76. Horrillo-Martínez, P.; Hultzsch, K. C. Tetrahedron Lett. 2009, 50 , 2054-2056. doi:10.1016/j.tetlet.2009.02.069

77. Zi, G. J. Organomet. Chem. 2010, 696, 68-75. doi:10.1016/j.jorganchem.2010.07.034

\section{License and Terms}

This is an Open Access article under the terms of the Creative Commons Attribution License (http://creativecommons.org/licenses/by/2.0), which permits unrestricted use, distribution, and reproduction in any medium, provided the original work is properly cited.

The license is subject to the Beilstein Journal of Organic Chemistry terms and conditions:

(http://www.beilstein-journals.org/bjoc)

The definitive version of this article is the electronic one which can be found at: doi:10.3762/bjoc.9.261 